\title{
CMS Billing Guidelines and Student Documentation: a New Era or New Burden?
}

\author{
Andre Kumar, MD and Jeffrey Chi, MD \\ Department of Medicine, Stanford University School of Medicine, Stanford, CA, USA.
}

\begin{abstract}
The Centers for Medicare and Medicaid Services (CMS) recently revised their Medicare Claims Processing Manual with the addition of CR 10412, a provision that permits teaching providers to fully bill for medical student notes. This change will have significant implications on the documentation duties of teaching physicians and trainees. Potential benefits of this provision include reduced documentation burden on house officers, improved medical student empowerment, and the infusion of more original content into the electronic medical record. However, these benefits may be offset by shifting the burden of documentation onto medical students, which may compromise their time spent with patients and overall wellness. In this perspective, we review the changes that occurred with CR 10412 and their potential impact on documentation across the medical education spectrum.
\end{abstract}

KEY WORDS: medical education; direct patient care; resident; training; electronic medical record.

J Gen Intern Med 34(4):634-5

DOI: $10.1007 /$ s11606-019-04853-7

(C) Society of General Internal Medicine 2019

$\mathrm{T}$ he Centers for Medicare and Medicaid Services (CMS) recently revised their Medicare Claims Processing Manual with the addition of CR 10412, a provision that permits attendings to fully bill for medical student notes. ${ }^{1}$ This change will have significant implications on the documentation duties of teaching physicians and trainees, especially if major insurance providers choose to follow this practice. In this perspective, we review the changes that occurred with CR 10412 and their potential impact on documentation.

Previously, providers were allowed to bill CMS for student documentation of the past medical/surgical history, family history, social history, and review of systems. Components of the student note that addressed Evaluation and Management (E/M) services, such as the physical examination or plan of care, required separate documentation by either a house officer or attending physician. CR 10412 now permits providers to bill CMS for student documentation of $\mathrm{E} / \mathrm{M}$ services in

Received September 12, 2018

Revised November 20, 2018

Accepted January 22, 2019

Published online February 12, 2019 addition to the historical items mentioned above. ${ }^{1}$ Providers are required to verify all student documentation and independently perform a history and physical examination, although explicit documentation of these activities outside of the student note are no longer required. Practically speaking, this updated policy negates the need for a house officer to generate a separate note for billing purposes. CR 10412 also keeps in place the "Primary Care Exception," whereby teaching physicians at properly accredited primary care practices are not required to directly interact with a patient to bill for services provided by a supervised resident for encounters of low- or mid-tier complexity. ${ }^{1}$

It is important to consider the previous state of documentation and billing that may have prompted this change. Previous requirements often resulted in documentation duplication, whereby both the medical student and house officer were required to write separate notes. Teaching providers, especially those overwhelmed by the complexity of the electronic health record (EHR), might have been more inclined to review house officer documentation as that was more pertinent for billing. Some providers might have neglected student documentation entirely, or considered it extraneous, resulting in missed opportunities to provide students with guidance on effective documentation practices. Multiple team members documenting in the EHR contributed to note bloat and potentiated chart lore if there were significant discrepancies between the student and house officer notes. This was especially deleterious during transitions of care which required an accurate representation of a patient's problems (such as inpatient discharge).

Time-motion studies have revealed that trainees spend a considerable amount of time in the EHR, up to triple the time spent with patients. ${ }^{2,3}$ Less than $15 \%$ of a trainee's time is spent in direct patient care. ${ }^{4}$ There are many reasons for these observations, but we cannot deny the burden of EHR documentation plays a significant role. It has altered the meaning of our work as physicians as well as our behavior. ${ }^{3,5}$ To avoid this burden and circumvent the need to write a separate note, house officers may copy and paste from student notes, instruct students to send drafts of their note via e-mail, or even have students use their login credentials to document under the resident's name.

Upon first glance, CR 10412 may be the solution to many of these challenges. Increasing the importance of student documentation may improve student empowerment while simultaneously allowing busy house officers to focus a greater portion 
of their time on direct patient care. It may also increase the likelihood that student notes will be read by supervising physicians such that timely feedback can be provided. However, it is ironic to ask the least experienced member of the team to play a potentially larger role in documentation, especially since the EHR can have a negative impact on physician well-being. ${ }^{6}$ Furthermore, CR10412 has the potential to compromise student learning and time spent with patients. It is not far-fetched to imagine scenarios of abuse whereby house officers experiencing burnout might relegate all documentation responsibilities onto the student. Students may be become less inclined to write accurate or thoughtful notes if the documentation burden is placed upon on them, a phenomenon already observed in intern physicians. ${ }^{7}$

It is unclear how widely adopted these practices will become, or if other institutions will modify their documentation policies to match the CMS regulations. If this occurs, one can argue these changes will infuse the chart with more original content from greater medical student contributions, but it is also possible the students will role model the documentation practices of near-peer residents: a status quo of note templates, auto-population, and copy forwarding. Documentation is important, and it fosters an opportunity for deeper reflection and learning. When done properly, it serves as a vital communication tool between care teams and provides a complete medical record that promotes effective healthcare delivery and quality. However, CR 10412 may force students to shoulder an increasing burden of documentation for the sake of billing throughput and convenience. It is quite possible we are about to offload our collective responsibilities onto our most vulnerable learners, while simultaneously convincing ourselves that it will improve trainee education and well-being.

Corresponding Author: Andre Kumar, MD; Department of Medicine, Stanford University School of Medicine, 300 Pasteur Dr. Stanford, Stanford, CA 94305, USA (e-mail: Akumar3@stanford.edu).

\section{Compliance with Ethical Standards:}

Conflict of Interest: The authors declare that they do not have a conflict of interest.

Publisher's Note: Springer Nature remains neutral with regard to jurisdictional claims in published maps and institutional affiliations.

\section{REFERENCES}

1. https://www.cms.gov/Outreach-and-Education/Medicare-Learning-Network-MLN/MLNMattersArticles/Downloads/MM10412.pdf (accessed December 11, 2018).

2. Ouyang $\mathbf{D}$, Chen JH, Hom $\mathbf{J}$ et al. Internal Medicine Resident Computer Usage: An Electronic Audit of an Inpatient Service. JAMA Intern Med. 2016;176(2):252-254.

3. Block L, Habicht R, Wu AW et al. In the Wake of the 2003 and 2011 Duty Hours Regulations, How Do Internal Medicine Interns Spend Their Time? J Gen Intern Med. 2013; 28(8):1042-1047.

4. Desai SV, Asch DA, Bellini LM et al. Educational outcomes in a dutyhour flexibility trail in internal medicine. $N$ Engl $J$ Med. 2018;378(16):1594-1950.

5. Rosenthal DI and Verghese A. Meaning and the Nature of Physicians' Work. N Engl J Med. 2016;375(19):1813-1815.

6. Shanafelt TD, Dyrbye LN, West CP. Addressing Physician Burnout: The Way Forward. JAMA. 2017;317(9):901-902.

7. Wang MD, Khanna R, Najafi N. Characterizing the Source of Text in Electronic Health Record Progress Notes. JAMA Intern Med. 2017;177(8): 1212-1213. 